\title{
Effects of a 12-Week Hatha Yoga Intervention on Cardiorespiratory Endurance, Muscular Strength and Endurance, and Flexibility in Hong Kong Chinese Adults: A Controlled Clinical Trial
}

\author{
Caren Lau, Ruby Yu, and Jean Woo \\ Department of Medicine and Therapeutics, The Chinese University of Hong Kong, Sha Tin, Hong Kong \\ Correspondence should be addressed to Ruby Yu; rubyyu@cuhk.edu.hk
}

Received 20 November 2014; Revised 15 March 2015; Accepted 18 March 2015

Academic Editor: Mariangela Rondanelli

Copyright (C) 2015 Caren Lau et al. This is an open access article distributed under the Creative Commons Attribution License, which permits unrestricted use, distribution, and reproduction in any medium, provided the original work is properly cited.

\begin{abstract}
Objective. To examine the effects of a 12-week Hatha yoga intervention on cardiorespiratory endurance, muscular strength and endurance, and flexibility in Chinese adults. Methods. 173 adults (aged $52.0 \pm 7.5$ years) were assigned to either the yoga intervention group $(n=87)$ or the waitlist control group $(n=86) .19$ dropped out from the study. Primary outcomes were changes in cardiorespiratory endurance (resting heart rate $(\mathrm{HR})$ and maximal oxygen uptake $\left(\mathrm{VO}_{2 \max }\right)$ ), muscular strength and endurance (curl-up and push-up tests), and lower back and hamstring flexibility (the modified back-saver sit-and-reach (MBS) test). Results. Compared to controls, the yoga group achieved significant improvements in $\mathrm{VO}_{2 \max }(P<0.01)$, curl-up $(P<0.05)$ and push-up $(P<0.001)$ tests, and the MBS left and right leg tests (both $P<0.001)$ in both genders. Significant change was also found for resting HR between groups in women $(P<0.05)$ but not in men. Further analysis comparing participants between younger and older subgroups yielded similar findings, except that the older participants in the yoga group failed to improve resting HR or the curl-up test versus control. Adherence (89\%) and attendance (94\%) were high. No serious adverse events occurred. Conclusion. A 12-week Hatha yoga intervention has favorable effects on cardiorespiratory endurance, muscular strength and endurance, and flexibility in Chinese adults.
\end{abstract}

\section{Introduction}

The health benefits of enhancing physical fitness (i.e., cardiorespiratory fitness (CRF), muscular fitness, and flexibility) have become well established during the past decades. Higher levels of CRF and muscular fitness are associated with significantly lower risk of developing metabolic syndrome $[1$, 2] and all-cause and cardiovascular mortality [3-7]. Although physical fitness declines as part of the physiological changes with age [8-10], the rate of decrease and possible reversibility might be amendable by intervention. Accumulating evidence indicates that an active lifestyle helps preserve CRF [11]. Furthermore, there is evidence around the benefits of aerobic and resistance exercises to improve CRF, muscular fitness, and health-related factors [12-14], thus highlighting the importance of intervention modalities. However, a large proportion of adults are much less active than desired [1517], where the major barriers include physical limitations, low self-efficacy, or simple aversion to exercise [18-20].

Increasing evidence suggests that complementary and alternative approaches that encourage increased physical activity and reduce sedentary behaviours might confer health benefits. Originated in India, yoga has become increasingly popular in western countries [21] as a means of exercise primarily using gentle static stretching postures with minimal physical exertion and conscious breathing to promote flexibility and relaxation. Of the various branches of yoga (such as Hindu, Hatha, Raja, and Mantra), Hatha yoga is perhaps the most widely practiced, which consists of elements of physical postures, conscious breathing, and meditation [22]. Hatha yoga appears safe and easy to learn and does not require any complicated or expensive equipment or specific training 


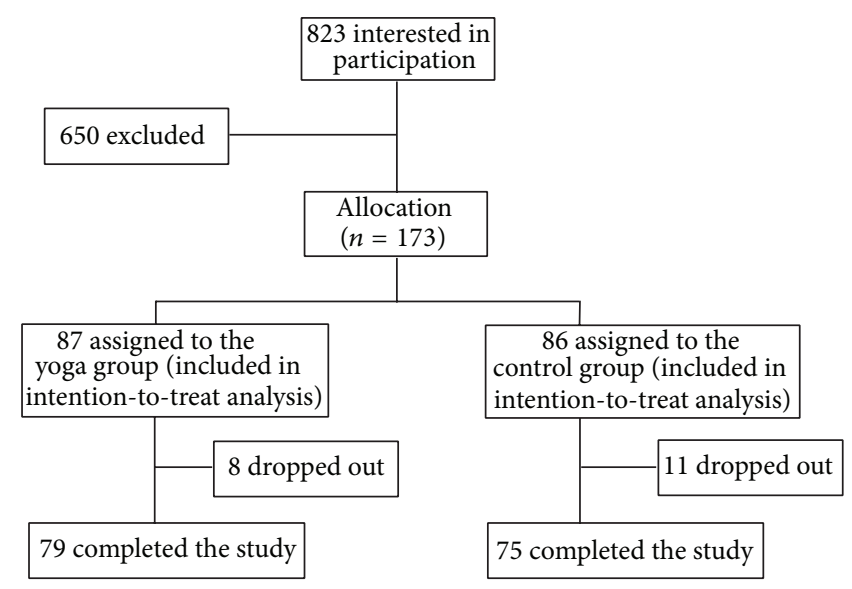

FIGURE 1: Recruitment of participants.

venue and thus could be suggested as an alternative form of exercise associated with high exercise adherence [23].

In addition to the beneficial effects on flexibility and relaxation, some yoga postures may achieve the recommended level of intensity for cardiovascular fitness [24, 25]. A growing number of research studies have shown that Hatha yoga can lead to improvements in CRF and muscular strength [26-29]. A recent review provides preliminary evidence of improvements in strength, balance, aerobic fitness, and selfrated health after yoga practice [30]. These benefits may be particularly important for people who are unable or unwilling to participate regularly in aerobic or resistance exercises. However, studies on the effects of yoga on CRF and muscular fitness have been few and have involved a small number of participants. The effects of yoga on physical fitness in Chinese adults have not been reported.

To fill these knowledge gaps, we developed a 12-week Hatha yoga intervention in the community to evaluate the effects of the intervention on cardiorespiratory endurance, muscular strength and endurance, and flexibility in a two-arm 12-week, prospective, nonblinded controlled trial enrolling Hong Kong Chinese adults. We also documented intervention adherence, attendance, and acceptability.

\section{Materials and Methods}

2.1. Participants. 173 Chinese men and women aged 18 and above were recruited for the study between May 2010 and January 2012. Recruitment was done by newspaper advertising, by placing notices in community centers, and by Internet publicity (including emails, advertisement, discussion forums, and website). Participants were volunteers, and the aim was to recruit a stratified sample so that similar proportions of males and females were obtained. An enrolment form was used for the screening purposes. Chinese individuals aged 18 and older, able to communicate in Cantonese, and physically and mentally capable of practicing yoga safely were included. Those who had severe medical conditions which limit their abilities to complete the whole course of treatment were excluded. In addition, those who were concurrently participating in yoga, qigong, meditation or other research studies were excluded.

2.2. Procedure. The study had adopted a prospective twoarm nonblinded controlled design. Each participant was individually assessed and they were grouped as 87 for yoga and 86 for control groups (Figure 1). To ensure that the proportions of male/female in the yoga and control groups would be similar and comparable for subgroup analysis, quota sampling was adopted, with gender used as quota control. Demographics, medication use, lifestyle factors, health-related quality of life (HRQoL), body measurements, and a battery of health-related physical fitness measures (described below) were taken before and after the 12-week protocol. Baseline assessments were performed between July 2010 and January 2012. Follow-up assessments were performed between October 2010 and May 2012. The study was conducted within a university-affiliated hospital (Prince of Wales Hospital, Sha Tin, New Territories, Hong Kong). All eligible participants participated voluntarily and their written informed consent was obtained prior to the study. The study was conducted as per the tenets of the Declaration of Helsinki with approval from the Joint Chinese University of Hong Kong-New Territories East Cluster Clinical Research Ethics Committee (registration number: CRE-2010.115; date of approval: 27 April 2010). The trial has been retrospectively registered in the Australian New Zealand Clinical Trials Registry (registration number: ACTRN12613000816752). The authors confirm that all ongoing and related trials for this intervention are registered.

2.3. Yoga Training. The yoga group participants were invited to attend a yoga training program consisting of 12 weekly 60-minute sessions, which were conducted by an Experienced Registered Yoga Teacher (E-RYT) (Yoga Alliance) with more than four years of Hatha yoga instructing experience. Throughout the 12 training sessions, participants were arranged in groups of seven to ten and were taught the breathing technique and 57 yogic poses commonly taught in community fitness centres including (1) standing poses which include Chair Pose (Utkatasana) and its 
variation (i.e., Chair with Torso Twist), Extended HandToe Pose (Utthita Hasta Padangusthasana), Extended Side Angle Pose (Utthita Parsvakonasana), Half Moon (Ardha Chandrasana), Modified Half Moon, Intense Side Stretch (Parsvottanasana), King of the Dancers Pose (Natarajasana), Lunge, Mountain Pose (Tadasana), Revolved Side Angle Pose (Parivrtta Parsvakonasana), Revolved Lunge, Revolved Triangle Pose (Parivrtta Trikonasana), Squat-Sitting-Down Pose (Malasana), Standing Forward Bend (Uttanasana), Tree Pose (Vrkshasana), Triangle Pose (Trikonasana), Warrior I (Virabhadrasana I), Warrior II (Virabhadrasana II), Warrior III (Virabhadrasana III), and Wide-Stance Forward Bend (Prasarita Padottanasana) and its variation (i.e., Wide-Stance Forward Bend with Torso Twist), (2) sitting poses which include Boat Pose (Navasana), Bound Angle Pose (Baddha Konasana), Cow-Face Pose (Gomukhasana), Half Load of the Fishes Pose (Ardha Matsyendrasana), Head-to-Knee Pose (Janu Shirshasana), Marichi I (Marichyasana I), Pigeon Pose (Rajakapotasana), Revolved Head-to-Knee Pose (Parivrtta Janu Shirshasana), Seated Forward Bend (Paschimottanasana), and Seated Wide-Angle Pose (Upavistha Konasana), (3) kneeling poses which include Camel Pose (Ushtrasana), Child's Pose (Balasana), Gate Pose (Parighasana), and One-Legged Royal Pigeon and Folded Forward (Rajakapotasana), (4) supine which include Apana Pose (Apanasana), Belly Twist (Jathara Parivartanasana), Bridge Pose (Setu Bandhasana) and its variation (i.e., Bridge with One Leg Lift), Corpse Pose (Shavasana), Preparation exercise for Plow Pose, Plow Pose (Halasana), Reclining Bound Angle Pose (Supta Baddha Konasana), Side-Reclining Leg Lift (Anantasana), and Supported Shoulder Stand (Salamba Sarvangasana), (5) prone poses which include Bow (Dhanurasana), Cobra (Bhujangasana), Locust (Shalabhasana), and Upward Facing Dog (Urdhva Mukha Svanasana), and (6) arm support poses which include Cat Cow (Durga Go) and its variation (i.e., Cat Cow with One Leg Lift), Four-Footed Table Top Pose (Chatus Pada Pitham), Plank (Utthita Hasta Padangusthasana), Table Top Exercise, Side Plank (Vasisthasana), and Upward Plank (Purvottanasna). Participants were also encouraged to practice yoga at home between classes (with handouts of yogic poses), and self-practice log sheets were used. Apart from the yoga program, participants in the yoga group were advised to maintain their routine activities and not to begin other exercise or mind-body program during the course of the study.

2.4. Control Group. The control group participants were requested to maintain their routine activities and not to begin any exercise, yoga, or mind-body program during the course of the study. To ensure adherence of the protocol, the control group participants also received the yoga program after the end of the study period.

\subsection{Measurements}

2.5.1. Cardiorespiratory Endurance. Resting heart rate (HR) was measured following a seated ten-minute rest period.
The HR was detected by electronic device (Polar Electro, Finland). Maximal oxygen uptake $\left(\mathrm{VO}_{2 \max }\right)$ was assessed with a maximal treadmill exercise test according to the Bruce protocol [31, 32]. Participants were instructed to abstain from any strenuous exercise on the day before testing. Each participant was connected to a calibrated respiratory gas analyzer (Fitmate, COSMED Srl, Italy) for gas analysis using a face mask. The respiratory gas analyzer was calibrated before each test. Treadmill speed was set initially at 1.7 miles per hour (mph) and 10\% grade. Every three minutes, the speed (and grade) increased to 2.5 (12\%), 3.4 (14\%), 4.2 (16\%), $5.0(18 \%)$, and finally $5.5(20 \%)$. Participants were verbally encouraged to reach their maximum. The test was terminated when the participant reached peak $\mathrm{VO}_{2}[33,34]$ or showed any symptoms that indicated termination of exercise based on the guidelines of the American College of Sports Medicine [32].

2.5.2. Muscular Strength and Endurance. The Canadian Standardized Test of Fitness (push-up and curl-up tests) was conducted to measure the muscular endurance of upper body muscles and the abdominal muscle groups, respectively. The test procedures for the measurements were according to the descriptions of Canadian Society for Exercise Physiology (CSEP) [32].

Push-Up Test. The participant started with the standard "down" position (the male participant was instructed to have hands pointing forward and under the shoulder, back straight, head up, using the toes as the pivotal point, whilst the female participant was instructed to have both legs together, lower leg in contact with mat with ankle plantar-flexed, back straight, hands shoulder width apart, head up, using the knees as the pivotal point). The participant raised the body by straightening the elbows and returning to the "down" position until the chin touches the mat and the abdomen should not touch the mat. The maximal number of push-ups performed consecutively without rest was counted as the score. The test was terminated when the participant strained forcibly or was unable to maintain the appropriate technique within two repetitions.

Curl-Up Test. The participant was instructed to perform a supine position on a mat with the knees at 90 degrees and place the hands on the thighs and curl up until the hands reach the knee caps. Shoes remain on during the test. The mobile metronome [35] was set to 50 beats per minute and the participant did slow, controlled curl ups to lift the shoulder blades off the mat (truck made 30-degree angle with the mat) in time with the metronome at a rate of 25 per minute. The test was conducted for one minute. The lower back had to be kept flattened before curling up. The participant was encouraged to perform as many curl-ups as possible without pausing, to a maximum of 25 [32].

2.5.3. Flexibility. The modified back-saver sit-and-reach (MBS) test was used to test lower back and hamstring flexibility [36]. The participant was requested to sit on a $30 \mathrm{~cm}$ 
high bench with one leg extended and resting on the bench, whilst the foot of the other leg was placed on the floor. A meter rule was placed on the bench between the legs and the heel of the extended leg was in line with the $50 \mathrm{~cm}$ level on the meter rule. The participant was required to stretch both arms out in front of the body with hands held together and fingers pointing toward the extended leg and was reminded to reach as far forward as possible without causing pain in the extended leg. The maximum distance that the tips of the middle fingers of both hands reached, as read from the meter rule, was indicated as the score of lower back and hamstring flexibility. Three trials were conducted with each leg and the maximum score for each leg (to the nearest $\mathrm{mm}$ ) was recorded and entered for analysis [37]. The MBS test was a comparatively better test to measure the lower back and hamstring flexibility than other protocols as its similarity of criterion-related validity in women but it had better criterionrelated validity in men, more practical, as it required minimal preparation time and equipment. It also eliminated excessive posterior compression of the vertebral disk when performing a single leg reach [36].

2.5.4. Other Covariates. Information on a number of covariates was also collected. Age, gender, marital status, education level, occupation, medication use, smoking, alcohol intake, physical activity level (assessed with the International Physical Activity Questionnaire, IPAQ [38, 39]), HRQoL (assessed with the Medical Outcomes Study (MOS) 36-item Short-Form Health Survey, SF-36 [40-42]), systolic blood pressure (SBP), diastolic blood pressure (DBP), and body mass index (BMI) were obtained by questionnaire interviews and measurements.

2.6. Data Processing and Analysis. We analysed the outcome variables in the intention-to-treat (ITT) population consisting of participants who completed the study protocol and those who completed the baseline assessment but dropped out from the study afterward. We used carryforward imputation to estimate the missing follow-up data in ITT population. Continuous and categorical variables were summarized as mean (SD) or by counts and percentages. We checked the homogeneity of the yoga and control groups with independent $t$-test and Mann-Whitney $U$ test for continuous variables and Chi-square $\left(\chi^{2}\right)$ test for categorical variables. Relationships between baseline characteristics and the outcome variables were determined with correlations. By incorporating findings from homogeneity check and correlations, possible covariates were identified. We tested the mean difference of each outcome variable between the yoga and control groups with analysis of variance (ANOVA) or analysis of covariance (ANCOVA) when possible covariates were identified. To assess the magnitude and direction of the effect of the yoga intervention relative to the control condition for each outcome variable, effect sizes were computed, where the value of partial eta-squared $\left(\eta^{2}\right)$ is represented as very small $(<0.01)$, small (0.01-0.05), medium (0.06-0.13), and large $(\geq 0.14)$. Covariates included BMI and DBP as well as HRQoL role emotional domain score and mental health component score. Men and women were initially analysed separately in statistical tests and the analyses were repeated in younger and older participants (below or above the median of the age distribution, i.e., 53.0 years) with both genders combined due to the small sample size of each subgroup. All statistical tests were two-tailed and the acceptance level of statistical significance $(P$ value $)$ in overall analysis was 0.05 or less. All statistical analyses were carried out using Windowsbased Statistical Package for the Social Sciences version 21.0 software (SPSS, Chicago, IL, USA).

\section{Results}

3.1. Baseline Characteristics. A total of 823 individuals were screened for eligibility and 66 were excluded due to health conditions. 39 prospective participants declined participation (reasons included their failure to make themselves available for attending the assessments or yoga classes) and 34 could not be contacted after multiple call attempts. Among the 684 prospective participants, 173 men $(n=64)$ and women $(n=$ 109) were recruited and assigned to either the yoga group $(n=$ 87 ) or the control group $(n=86)$, of which 19 dropped out from the study.

The baseline characteristics of participants are shown in Table 1. The mean age was $52.0 \pm 7.5$ (31.0-71.0) years. Most of the participants were married (81.5\%) and had secondary education (93.6\%). About half engaged in full-time jobs $(53.8 \%)$. Since the two genders differed significantly in various baseline characteristics and the outcome measures, subsequent analyses were undertaken separately for men and women.

In comparing the yoga and the control group, in both genders, there were no significant differences in baseline characteristics except that, in men, the yoga group had lower flexibility (MBS leg and right leg tests, both $P<0.01$ ), whereas in women, the yoga group had lower HRQoL role emotional domain score and BMI (both $P<0.05$, Table 2 ).

3.2. Effects of Intervention on Outcome Measures. In men, the yoga group achieved greater increase in $\mathrm{VO}_{2 \max }$ than the control group $(P<0.01)$. Significant improvements were also found for muscular strength and endurance in the yoga group compared with the control group (curl-up test $P<$ 0.05 , push-up test $P<0.001$ ). Lower back and hamstring flexibility increased significantly in the yoga group but not in the control group (MBS both left and right leg tests $P<$ 0.001). No significant differences were found for resting HR between groups $(P=0.086)$. In women, the yoga group also achieved greater increase in $\mathrm{VO}_{2 \max }(P<0.01)$, the curlup $(P<0.05)$ and push-up $(P<0.001)$ tests as well as the MBS left and right leg tests (both $P<0.001$ ) than the control group. Significant improvement was also found for resting $\mathrm{HR}$ in the yoga group compared to the control group $(P<0.05$, Table 3$)$.

The analyses were repeated in younger and older participants, with both genders combined. In younger participants, the yoga group achieved greater improvements in resting HR $(P<0.05), \mathrm{VO}_{2 \max }(P<0.01)$, the curl-up $(P<0.01)$, 
TABLE 1: Demographic characteristics of participants, by gender ( $N$ $=173)$.

\begin{tabular}{|c|c|c|c|}
\hline Characteristics & $\begin{array}{c}\text { Men } \\
(n=64) \\
\quad \mathrm{M} \pm \mathrm{SD}\end{array}$ & $\begin{aligned} & \text { Women } \\
&(n=109) \\
& \text { or } f(\%)\end{aligned}$ & $P$ \\
\hline Age, years & $53.59 \pm 8.02$ & $51.04 \pm 6.97$ & 0.029 \\
\hline \multicolumn{4}{|l|}{ Marital status, $\%^{\dagger}$} \\
\hline Single & $2(3.1)$ & $25(22.9)$ & 0.050 \\
\hline Married & $57(89.1)$ & $84(77.1)$ & \\
\hline Widowed & $5(7.8)$ & $0(0)$ & \\
\hline Education level, $\%^{\dagger}$ & & & $<0.001$ \\
\hline No education & $0(0)$ & $1(0.9)$ & \\
\hline Primary & $0(0)$ & $10(9.2)$ & \\
\hline Secondary & $29(45.3)$ & $70(64.2)$ & \\
\hline Tertiary & $35(54.7)$ & $28(25.7)$ & \\
\hline Occupation, $\%^{\dagger}$ & & & 0.973 \\
\hline Full-time & $37(57.8)$ & $56(51.4)$ & \\
\hline Part-time & $1(1.6)$ & $9(8.3)$ & \\
\hline Unemployed & $2(3.1)$ & $0(0)$ & \\
\hline Housewife & $0(0)$ & $30(27.5)$ & \\
\hline Retired & $24(37.5)$ & $14(12.8)$ & \\
\hline \multicolumn{4}{|l|}{ Medication use, \% } \\
\hline Antidiabetic & & & 0.489 \\
\hline Yes & $10(15.6)$ & $13(11.9)$ & \\
\hline No & $54(84.4)$ & $96(88.1)$ & \\
\hline Antihypertensive & & & 0.118 \\
\hline Yes & $14(21.9)$ & $36(33.0)$ & \\
\hline No & $50(78.1)$ & $73(67.0)$ & \\
\hline Lipid lowering & & & 0.146 \\
\hline Yes & $6(9.4)$ & $19(17.4)$ & \\
\hline No & $58(90.6)$ & $90(82.6)$ & \\
\hline Smoking, $\%^{\dagger}$ & & & $<0.001$ \\
\hline Current & $3(4.7)$ & $0(0)$ & \\
\hline Quitted & $10(15.6)$ & $1(0.9)$ & \\
\hline Never & $51(79.7)$ & $108(99.1)$ & \\
\hline Alcohol intake, $\%^{\dagger}$ & & & 0.004 \\
\hline Quitted & $2(3.1)$ & $0(0)$ & \\
\hline Never & $18(28.1)$ & $55(50.5)$ & \\
\hline Sometimes & $40(62.5)$ & $52(47.7)$ & \\
\hline Always & $4(6.3)$ & $2(1.8)$ & \\
\hline $\begin{array}{l}\text { Physical activity level, } \\
\text { MET-minutes/week }\end{array}$ & $\begin{array}{l}1450.86 \pm \\
918.85\end{array}$ & $\begin{array}{c}1936.63 \pm \\
1926.52\end{array}$ & 0.041 \\
\hline \multicolumn{4}{|l|}{ HRQoL } \\
\hline Physical function (PF) & $91.09 \pm 10.96$ & $85.00 \pm 11.30$ & 0.001 \\
\hline Role physical (RP) & $88.67 \pm 23.12$ & $81.64 \pm 29.59$ & 0.085 \\
\hline Bodily pain (BP) & $70.55 \pm 21.35$ & $64.52 \pm 20.70$ & 0.065 \\
\hline $\begin{array}{l}\text { General health } \\
\text { perceptions (GH) }\end{array}$ & $60.16 \pm 11.41$ & $61.24 \pm 12.25$ & 0.566 \\
\hline $\begin{array}{l}\text { Physical component } \\
\text { score (PCS) }\end{array}$ & $310.47 \pm 47.30$ & $292.31 \pm 56.03$ & 0.031 \\
\hline Vitality (VT) & $69.14 \pm 13.99$ & $64.45 \pm 16.82$ & 0.050 \\
\hline Social functioning (SF) & $87.89 \pm 14.26$ & $85.21 \pm 18.72$ & 0.290 \\
\hline Role emotional (RE) & $85.42 \pm 29.02$ & $78.59 \pm 34.10$ & 0.164 \\
\hline $\begin{array}{l}\text { General mental Health } \\
(\mathrm{MH})\end{array}$ & $78.06 \pm 12.75$ & $75.71 \pm 14.74$ & 0.288 \\
\hline
\end{tabular}

TABle 1: Continued.

\begin{tabular}{|c|c|c|c|}
\hline Characteristics & $\begin{array}{l}\text { Men } \\
\begin{array}{l}(n=64) \\
\mathrm{M} \pm \mathrm{SD}\end{array}\end{array}$ & $\begin{array}{r}\text { Women } \\
(n=109) \\
\text { or } f(\%)\end{array}$ & $P$ \\
\hline $\begin{array}{l}\text { Mental component score } \\
\text { (MCS) }\end{array}$ & $320.51 \pm 53.10$ & $\begin{array}{c}303.96 \pm \\
70.87\end{array}$ & 0.083 \\
\hline \multicolumn{4}{|l|}{ Body measurements } \\
\hline $\mathrm{SBP}, \mathrm{mmHg}$ & $132.52 \pm 18.21$ & $129.11 \pm 18.69$ & 0.244 \\
\hline $\mathrm{DBP}, \mathrm{mmHg}$ & $79.56 \pm 11.01$ & $78.57 \pm 11.41$ & 0.576 \\
\hline BMI, $\mathrm{kg} / \mathrm{m}^{2}$ & $24.83 \pm 3.65$ & $25.37 \pm 4.08$ & 0.388 \\
\hline \multicolumn{4}{|l|}{ Outcome measures } \\
\hline \multicolumn{4}{|l|}{$\begin{array}{l}\text { Cardiorespiratory } \\
\text { endurance }\end{array}$} \\
\hline $\begin{array}{l}\text { Resting heart rate, } \\
\text { bpm }\end{array}$ & $67.17 \pm 9.14$ & $69.88 \pm 9.53$ & 0.069 \\
\hline \multicolumn{4}{|l|}{$\begin{array}{l}\text { Muscular strength and } \\
\text { endurance }\end{array}$} \\
\hline Curl-ups, times & $21.25 \pm 5.50$ & $20.18 \pm 5.19$ & 0.204 \\
\hline Push-ups, times & $8.17 \pm 8.09$ & $1.84 \pm 3.83$ & $<0.001$ \\
\hline \multicolumn{4}{|l|}{ Flexibility } \\
\hline MBS left leg, cm & $44.34 \pm 13.15$ & $51.85 \pm 9.62$ & $<0.001$ \\
\hline MBS right leg, $\mathrm{cm}$ & $44.30 \pm 13.71$ & $51.63 \pm 9.89$ & $<0.001$ \\
\hline
\end{tabular}

The total percentage may not add up exactly to $100 \%$ due to rounding. BMI: body mass index; DBP: diastolic blood pressure; HRQoL: healthrelated quality of life; MBS: the modified back-saver sit-and-reach test; SBP: systolic blood pressure; $\mathrm{VO}_{2 \max }$ : maximal oxygen uptake.

${ }^{\dagger}$ Variables were regrouped for analyses as follows: marital status (single + widowed versus married), education level (No education + primary + secondary versus tertiary), occupation (full-time + part-time versus unemployed + housewife + retired), smoking (current + quitted versus never), and alcohol intake (quitted + sometimes + always versus never).

and push-up $(P<0.001)$ tests as well as the MBS left and right leg tests (both $P<0.001$ ) than the control group. In older participants, the yoga group also achieved greater improvements in $\mathrm{VO}_{2 \max }(P<0.01)$, the push-up test $(P<$ 0.001 ), and the MBS left and right leg tests (both $P<0.001$ ) than the control group. However, no significant differences were found for resting $\operatorname{HR}(P=0.128)$ and the curl-up test $(P=0.103)$ between groups (Table 4$)$.

3.3. Adherence, Attendance, and Acceptability. The overall adherence rate was $89 \%$ (yoga group: $92 \%$, control group: $87 \%$ ) and the mean attendance rate of 12 sessions was $94 \%$. According to the log records for practice at home, $99 \%$ of the yoga participants practiced yoga at home for an average of about 165 minutes/week (23 minutes/day). Qualitative feedback indicated that the intervention was well received, with most of the participants reporting improvements in health after yoga participation. While some of the participants reported some difficulties with the yoga postures at the beginning of the intervention, no unanticipated adverse events were reported.

\section{Discussion}

The present study was conducted to examine the effects of a 12-week Hatha yoga on cardiorespiratory endurance, 
TABLE 2: Demographic characteristics of participants in the yoga group and the control group, by gender.

\begin{tabular}{|c|c|c|c|c|c|c|}
\hline \multirow{3}{*}{ Characteristics } & \multicolumn{3}{|c|}{ Men } & \multicolumn{3}{|c|}{ Women } \\
\hline & Yoga $(n=34)$ & Control $(n=30)$ & \multirow{2}{*}{$P$} & Yoga $(n=53)$ & Control $(n=56)$ & \multirow{2}{*}{$P$} \\
\hline & \multicolumn{2}{|c|}{$\mathrm{M} \pm \mathrm{SD}$ or $f(\%)$} & & $\mathrm{M} \pm \mathrm{SD}$ & or $f(\%)$ & \\
\hline Age, years & $53.68 \pm 7.91$ & $53.50 \pm 8.27$ & 0.931 & $51.64 \pm 6.57$ & $50.46 \pm 7.35$ & 0.381 \\
\hline Marital status, $\%^{\dagger}$ & & & 0.067 & & & 0.589 \\
\hline Single & $2(5.9)$ & $0(0)$ & & $11(20.8)$ & $14(25.0)$ & \\
\hline Married & $28(82.4)$ & $29(96.7)$ & & $42(79.2)$ & $42(75.0)$ & \\
\hline Widowed & $4(11.8)$ & $1(3.3)$ & & $0(0.0)$ & $0(0)$ & \\
\hline Education level, $\%^{\dagger}$ & & & 0.087 & & & 0.543 \\
\hline No education & $0(0)$ & $0(0)$ & & $0(0)$ & $1(1.8)$ & \\
\hline Primary & $0(0)$ & $0(0)$ & & $5(9.4)$ & $5(8.9)$ & \\
\hline Secondary & $12(35.3)$ & $17(56.7)$ & & $33(62.3)$ & $37(66.1)$ & \\
\hline Tertiary & $22(64.7)$ & $13(43.3)$ & & $15(28.3)$ & $13(23.2)$ & \\
\hline Occupation, $\%^{\dagger}$ & & & 0.355 & & & 0.813 \\
\hline Full-time & $21(61.8)$ & $16(53.5)$ & & $27(50.9)$ & $29(51.8)$ & \\
\hline Part-time & $1(2.9)$ & $0(0)$ & & $4(7.5)$ & $5(8.9)$ & \\
\hline Unemployed & $0(0)$ & $2(6.7)$ & & $0(0)$ & $0(0)$ & \\
\hline Housewife & $0(0)$ & $0(0)$ & & $18(34.0)$ & $12(21.4)$ & \\
\hline Retired & $12(35.3)$ & $12(40)$ & & $4(7.5)$ & $10(17.9)$ & \\
\hline \multicolumn{7}{|l|}{ Medication use, $\%$} \\
\hline Antidiabetic & & & 0.064 & & & 0.688 \\
\hline Yes & $8(23.5)$ & $2(6.7)$ & & $7(13.2)$ & $6(10.7)$ & \\
\hline No & $26(76.5)$ & $28(93.3)$ & & $46(86.8)$ & $50(89.3)$ & \\
\hline Antihypertensive & & & 0.791 & & & 0.066 \\
\hline Yes & $7(20.6)$ & $7(23.3)$ & & $13(24.5)$ & $23(41.1)$ & \\
\hline No & $27(79.4)$ & $23(76.7)$ & & $40(75.5)$ & $33(58.9)$ & \\
\hline Lipid lowering & & & 0.872 & & & 0.904 \\
\hline Yes & $3(8.8)$ & $3(10.0)$ & & $9(17.0)$ & $10(17.9)$ & \\
\hline No & $31(91.2)$ & $27(90.0)$ & & $44(83.0)$ & $46(82.1)$ & \\
\hline Smoking, $\%^{\dagger}$ & & & 0.953 & & & 0.328 \\
\hline Current & $1(2.9)$ & $2(6.7)$ & & $0(0)$ & $1(1.8)$ & \\
\hline Quitted & $6(17.6)$ & $4(13.3)$ & & $0(0)$ & $0(0)$ & \\
\hline Never & $27(79.4)$ & $24(80.0)$ & & $53(100)$ & $55(98.2)$ & \\
\hline Alcohol intake, $\%^{\dagger}$ & & & 0.384 & & & 0.069 \\
\hline Quitted & $1(2.9)$ & $1(3.3)$ & & $0(0)$ & $0(0)$ & \\
\hline Never & $8(23.5)$ & $10(33.3)$ & & $22(41.5)$ & $33(58.9)$ & \\
\hline Sometimes & $23(67.6)$ & $17(56.7)$ & & $30(56.6)$ & $22(39.3)$ & \\
\hline Always & $2(5.9)$ & $2(6.7)$ & & $1(1.9)$ & $1(1.8)$ & \\
\hline $\begin{array}{l}\text { Physical activity level, } \\
\text { MET-minutes/week }\end{array}$ & $1332.36 \pm 1047.76$ & $1569.36 \pm 769.31$ & 0.330 & $2109.24 \pm 2145.74$ & $1781.99 \pm 1714.95$ & 0.422 \\
\hline \multicolumn{7}{|l|}{ HRQoL } \\
\hline Physical function (PF) & $93.24 \pm 5.89$ & $88.67 \pm 14.50$ & 0.096 & $85.85 \pm 11.88$ & $84.20 \pm 10.78$ & 0.448 \\
\hline Role physical (RP) & $91.18 \pm 19.35$ & $85.83 \pm 26.82$ & 0.360 & $77.36 \pm 32.62$ & $85.71 \pm 26.05$ & 0.141 \\
\hline Bodily pain (BP) & $71.26 \pm 19.07$ & $69.73 \pm 23.99$ & 0.777 & $62.57 \pm 19.87$ & $66.18 \pm 21.49$ & 0.365 \\
\hline General health perceptions (GH) & $61.03 \pm 11.33$ & $59.17 \pm 11.60$ & 0.519 & $60.47 \pm 12.37$ & $61.96 \pm 12.20$ & 0.527 \\
\hline Physical component score (PCS) & $316.71 \pm 33.06$ & $303.40 \pm 59.33$ & 0.282 & $286.25 \pm 60.58$ & $298.05 \pm 51.23$ & 0.273 \\
\hline Vitality (VT) & $71.03 \pm 12.30$ & $67.00 \pm 15.62$ & 0.253 & $64.06 \pm 16.11$ & $64.82 \pm 17.61$ & 0.814 \\
\hline Social functioning (SF) & $86.40 \pm 13.89$ & $89.58 \pm 14.71$ & 0.376 & $83.25 \pm 20.35$ & $87.05 \pm 17.02$ & 0.292 \\
\hline Role emotional (RE) & $80.39 \pm 32.94$ & $91.11 \pm 23.05$ & 0.133 & $71.07 \pm 37.00$ & $85.71 \pm 29.72$ & 0.025 \\
\hline General mental Health $(\mathrm{MH})$ & $77.65 \pm 11.49$ & $78.53 \pm 14.23$ & 0.784 & $73.74 \pm 15.08$ & $77.57 \pm 14.30$ & 0.176 \\
\hline Mental component score (MCS) & $315.47 \pm 53.26$ & $326.23 \pm 53.23$ & 0.423 & $292.12 \pm 75.16$ & $315.16 \pm 65.25$ & 0.090 \\
\hline \multicolumn{7}{|l|}{ Body measurements } \\
\hline SBP, mmHg & $132.82 \pm 16.80$ & $132.17 \pm 19.97$ & 0.887 & $125.87 \pm 15.21$ & $132.18 \pm 21.16$ & 0.076 \\
\hline DBP, $\mathrm{mmHg}$ & $78.32 \pm 10.79$ & $80.97 \pm 11.27$ & 0.342 & $76.64 \pm 10.43$ & $80.39 \pm 12.07$ & 0.086 \\
\hline BMI, $\mathrm{kg} / \mathrm{m}^{2}$ & $24.28 \pm 4.21$ & $25.45 \pm 2.83$ & 0.203 & $24.55 \pm 3.62$ & $26.14 \pm 4.37$ & 0.041 \\
\hline
\end{tabular}


TABLE 2: Continued.

\begin{tabular}{|c|c|c|c|c|c|c|}
\hline \multirow{3}{*}{ Characteristics } & \multicolumn{3}{|c|}{ Men } & \multicolumn{3}{|c|}{ Women } \\
\hline & Yoga $(n=34)$ & Control $(n=30)$ & $P$ & Yoga $(n=53)$ & Control $(n=56)$ & $P$ \\
\hline & \multicolumn{2}{|c|}{$\mathrm{M} \pm \mathrm{SD}$ or $f(\%)$} & $P$ & \multicolumn{2}{|c|}{$\mathrm{M} \pm \mathrm{SD}$ or $f(\%)$} & $\boldsymbol{I}$ \\
\hline \multicolumn{7}{|l|}{ Outcome measures } \\
\hline \multicolumn{7}{|l|}{ Cardiorespiratory endurance } \\
\hline Resting heart rate, bpm & $67.53 \pm 9.31$ & $66.77 \pm 9.10$ & 0.742 & $69.11 \pm 9.06$ & $70.61 \pm 9.97$ & 0.416 \\
\hline $\mathrm{VO}_{2 \max }, \mathrm{mL} / \mathrm{kg} / \mathrm{min}$ & $32.99 \pm 6.77$ & $32.95 \pm 6.76$ & 0.982 & $26.35 \pm 5.51$ & $24.69 \pm 4.37$ & 0.083 \\
\hline \multicolumn{7}{|c|}{ Muscular strength and endurance } \\
\hline Curl-ups, times & $20.18 \pm 6.42$ & $22.47 \pm 4.00$ & 0.088 & $21.04 \pm 5.43$ & $19.38 \pm 4.86$ & 0.095 \\
\hline Push-ups, times & $9.50 \pm 9.50$ & $6.67 \pm 5.93$ & 0.153 & $1.87 \pm 4.30$ & $1.82 \pm 3.37$ & 0.950 \\
\hline \multicolumn{7}{|l|}{ Flexibility } \\
\hline MBS left leg, cm & $39.99 \pm 11.47$ & $49.28 \pm 13.36$ & 0.004 & $52.46 \pm 8.44$ & $51.28 \pm 10.66$ & 0.520 \\
\hline MBS right leg, cm & $39.66 \pm 11.80$ & $49.56 \pm 14.00$ & 0.003 & $52.41 \pm 8.31$ & $50.89 \pm 11.21$ & 0.424 \\
\hline
\end{tabular}

The total percentage may not add up exactly to $100 \%$ due to rounding.

BMI: body mass index; DBP: diastolic blood pressure; HRQoL: health-related quality of life; MBS: the modified back-saver sit-and-reach test; SBP: systolic blood pressure; $\mathrm{VO}_{2 \max }$ : maximal oxygen uptake.

${ }^{\dagger}$ Variables were regrouped for analyses as follows: marital status (single + widowed versus married), education level (No education + primary + secondary versus tertiary), occupation (full-time + part-time versus unemployed + housewife + retired), smoking (current + quitted versus never), and alcohol intake (quitted + sometimes + always versus never).

muscular strength and endurance, and lower back and hamstring flexibility in Hong Kong Chinese adults. Our results demonstrated significant improvements in $\mathrm{VO}_{2 \max }$, muscular strength, and flexibility in both men and women who practiced yoga compared to the control group. The yoga group also showed a reduction in resting HR compared to the control group in women. Further analysis comparing participants between younger and older subgroups yielded similar findings, except for the older participants in the yoga group, who failed to improve the resting HR or the curl-up test versus the control group.

Studies investigating the effects of yoga on cardiorespiratory endurance are limited. Sivasankaran et al. [43] showed that the resting HR was significantly reduced by 9 beats/minute after a 6-week program of yoga and meditation in people with and without coronary artery disease. Another study on the effects of yoga on resting HR and blood pressure in middle-aged adults was reported by Devasena and Narhare [44] who found that a 6-month yoga program (with 1-hour daily practice session) could significantly reduce resting HR over the course of the intervention. However, these two studies have been performed without a comparison group. Using a nonblinded controlled study design, the present study showed no benefit to men with regard to resting HR, but a modest effect was observed in reducing resting HR in women. The reduction observed in women may be mediated by the training-induced reduction of respiratory rate and improvement in the baroreflex sensitivity [45-47], attributed to the relaxation and breathing techniques adopted in yoga training [48]. The lack of significant improvement in resting $\mathrm{HR}$ in men does not necessarily imply that the yoga intervention is not beneficial to cardiorespiratory endurance but may relate to the relatively lower resting $\mathrm{HR}$ at baseline in men ( 67.17 beats/minute) compared with women ( 69.88 beats/minute), so that the effect of yoga regarding this measure in men was less pronounced than that in women. Our findings also demonstrated a significant improvement in resting HR in the younger but not in the older participants. This is in contrast to a previous yoga intervention conducted by Bowman et al. [49] who has reported a significant reduction in resting HR following a 6-week yoga program in elderly person aged 62-81 years. This disparity between studies could be attributed to many factors, including differences between intervention and population characteristics. Respiratory rate is a possible confounding factor. However, we have no such measurement in the study.

In addition to improving resting $\mathrm{HR}$, the yoga intervention increased $\mathrm{VO}_{2 \max }$ in both men and women compared with the control group. When comparing participants between younger and older subgroups, the yoga intervention was similarly effective in increasing $\mathrm{VO}_{2 \max }$ in both subgroups. Prior studies showed improved $\mathrm{VO}_{2 \text { peak }}$ for healthy untrained adults [26] and people with chronic heart failure who completed an 8-week yoga training program [50]. We add to these prior studies by showing an improved $\mathrm{VO}_{2 \max }$ for apparently healthy adults assigned to a 12-week yoga training program. While the mechanisms responsible for the change cannot be directly determined in this study, the increased muscular endurance resulting from yoga practice may have achieved a better control of intercostal muscles that would subsequently improve $\mathrm{VO}_{2 \max }$.

Previous studies have reported that yoga exercise improved truck dynamic muscular strength and endurance as well as abdominal muscles muscular strength [27, 29]. Similar data were observed with a significant improvement in lower limb muscular endurance in older adults [28]. Our study also demonstrated significant favorable effects of the yoga intervention on muscular strength and endurance of upper body muscles and abdominal muscles in both men and women. The mechanisms by which yoga provides beneficial effects on muscular strength and endurance still need to be determined but it is reasonable to believe that the benefit may be mediated by some yoga postures, which may have achieved optimal intensity for increasing muscular strength 


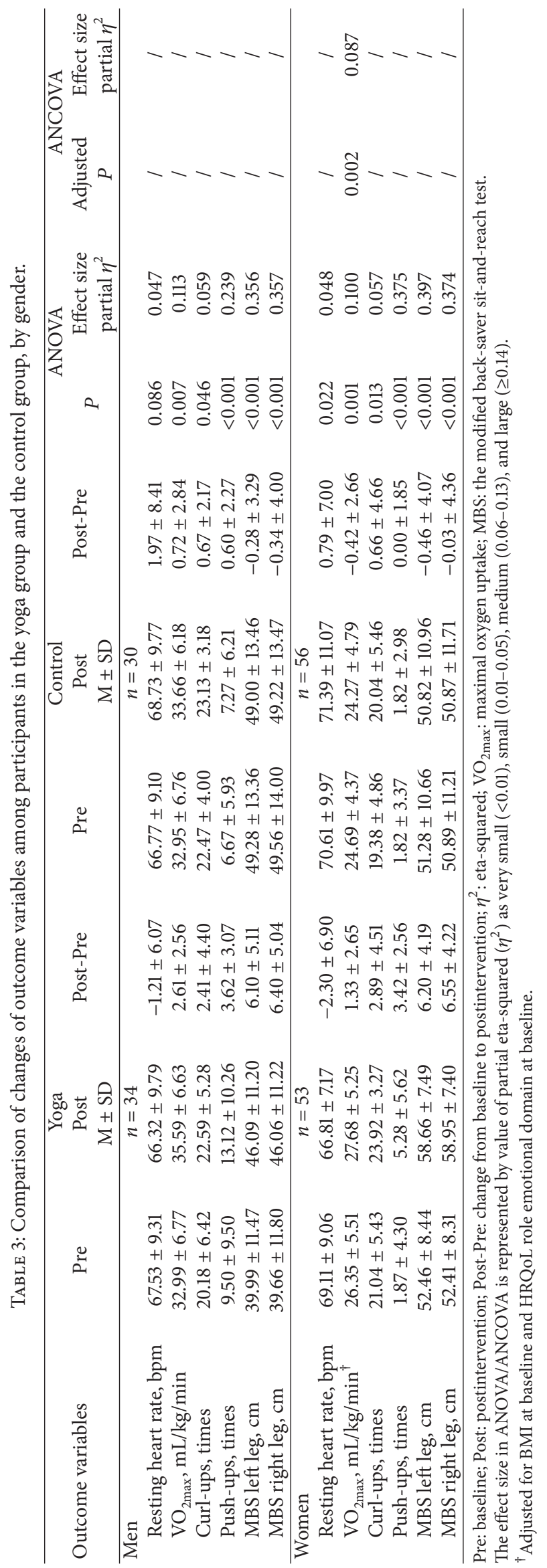




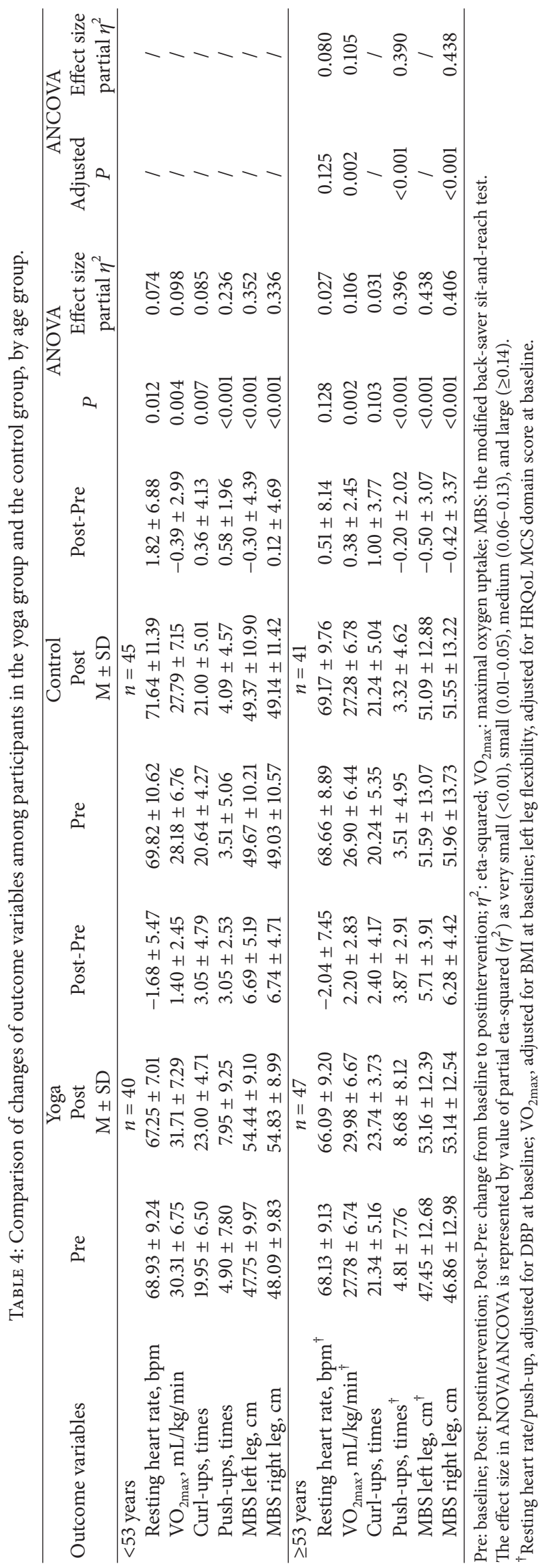


and endurance. A previous electromyographic (EMG) analysis in 20 older adults showed that some selected yoga postures including the Chair and Warrior Front postures had generated relatively large knee extensor joint moments of force and quadriceps EMG activity [51]. In addition, yoga may help to maintain proper posture and spinal alignment, thereby exerting beneficial effects on muscular strength and endurance. However, abdominal muscular strength failed to improve in the yoga group versus the control group within older participants. It is possible that the intensity of yoga was not sufficiently high to provide observable improvements in this measure in older adults who tend to have weak abdominal muscles. However, we did not measure muscle mass in this study.

An additional benefit of the yoga intervention was improvement in flexibility, which can determine the efficiency of muscles. Our findings demonstrated significant favorable effects of the yoga program on lower back and hamstring flexibility in both genders and age groups. These results are in accordance with previous research that found yoga significantly improved ankle flexibility, shoulder elevation, trunk extension, and trunk flexion in healthy untrained adults [26] and spinal flexibility in people with chronic low back pain [52]. The findings of the increased flexibility are not unexpected, given the static stretching nature of yoga postures (involving holding the stretched position using the strength of the agonist muscle) [26], which exert their beneficial effects on flexibility by increasing the length of both connective and muscle tissue [53], thus engendering a range of joint angles.

The feasibility of the yoga program was also demonstrated by (1) the low attrition rate (11.0\%), which was lower compared to overseas studies with similar study design (15.3$22.0 \%)[54,55],(2)$ the good attendance, with participants attending an average of $94 \%$ of available sessions, and (3) the positive feedback from participants, with all of them suggesting that the intervention was well received and most of them believing yoga is helpful for enhancing their health. Compliance was good, with most of the participants reported practicing yoga at home. Although most of the participants reported some difficulties with some of the yoga postures at the beginning of the intervention, class attendance was high. Therefore, our results suggest that Hatha yoga may be an alternative training modality for health enhancements.

The limitations of this study include the lack of randomization, which may have led to selection bias and decreased comparability between groups with various confounding factors. Other limitations include nonblinded assessment of outcomes and the possible lack of measures of confounding factors such as diet and sleep quality. In addition, the participants were a highly motivated group that was willing to volunteer for a research study, and the yoga class was adapted for beginners; therefore findings may not be directly generalizable to a typical community yoga class. Finally, because the yoga classes were offered to participants once per week, the frequency of sessions may be different from that of other intervention studies, which makes comparison between studies difficult. However, our study has several notable strengths including the use of an expert yoga practitioner to design a program specifically for middle-aged to older adults, a sex-balanced stratified sample, and the inclusion of multiple outcome variables.

In conclusion, this study showed that a 12-week Hatha yoga program produced beneficial changes in cardiovascular endurance, muscular strength and endurance, and flexibility for Chinese adults. Given the high adherence and attendance rates as well as the encouraging results of this study, yoga may be a promising alternative form of exercise. Further investigation with longer follow-up (e.g., 6 months) should be considered, which would offer insights as to the long-term benefits of yoga.

\section{Conflict of Interests}

The authors declare that they have no competing interests regarding the publication of this paper.

\section{Acknowledgments}

The authors would like to thank the study participants and staff in both Department of Medicine and Therapeutics and Centre for Nutritional Studies at the Chinese University of Hong Kong who gave generously of their time and effort.

\section{References}

[1] D. E. Laaksonen, H.-M. Lakka, J. T. Salonen, L. K. Niskanen, R. Rauramaa, and T. A. Lakka, "Low levels of leisure-time physical activity and cardiorespiratory fitness predict development of the metabolic syndrome," Diabetes Care, vol. 25, no. 9, pp. 1612-1618, 2002.

[2] R. Jurca, M. J. Lamonte, C. E. Barlow, J. B. Kampert, T. S. Church, and S. N. Blair, "Association of muscular strength with incidence of metabolic syndrome in men," Medicine \& Science in Sports and Exercise, vol. 37, no. 11, pp. 1849-1855, 2005.

[3] S. N. Blair, J. B. Kampert, H. W. Kohl III et al., "Influences of cardiorespiratory fitness and other precursors on cardiovascular disease and all-cause mortality in men and women," Journal of the American Medical Association, vol. 276, no. 3, pp. 205-210, 1996.

[4] S. W. Farrell, J. B. Kampert, H. W. Kohl III et al., "Influences of cardiorespiratory fitness levels and other predictors on cardiovascular disease mortality in men," Medicine \& Science in Sports \& Exercise, vol. 30, no. 6, pp. 899-905, 1998.

[5] A. B. Newman, V. Kupelian, M. Visser et al., "Strength, but not muscle mass, is associated with mortality in the health, aging and body composition study cohort," Journals of Gerontology Series A-Biological Sciences and Medical Sciences, vol. 61, no. 1, pp. 72-77, 2006.

[6] C. R. Gale, C. N. Martyn, C. Cooper, and A. A. Sayer, "Grip strength, body composition, and mortality," International Journal of Epidemiology, vol. 36, no. 1, pp. 228-235, 2007.

[7] S. Kodama, K. Saito, S. Tanaka et al., "Cardiorespiratory fitness as a quantitative predictor of all-cause mortality and cardiovascular events in healthy men and women: a meta-analysis," Journal of the American Medical Association, vol. 301, no. 19, pp. 2024-2035, 2009.

[8] J. L. Fleg, F. O’Connor, G. Gerstenblith et al., "Impact of age on the cardiovascular response to dynamic upright exercise in 
healthy men and women," Journal of Applied Physiology, vol. 78, no. 3, pp. 890-900, 1995.

[9] I. Janssen, S. B. Heymsfield, Z. Wang, and R. Ross, "Skeletal muscle mass and distribution in 468 men and women aged 1888 yr," Journal of Applied Physiology, vol. 89, no. 1, pp. 81-88, 2000.

[10] D. K. McGuire, B. D. Levine, J. W. Williamson et al., "A 30-year follow-up of the Dallas bed rest and training study: I. Effect of age on the cardiovascular response to exercise," Circulation, vol. 104, no. 12, pp. 1350-1357, 2001.

[11] J. R. Stofan, L. DiPietro, D. Davis, H. W. Kohl III, and S. N. Blair, "Physical activity patterns associated with cardiorespiratory fitness and reduced mortality: the Aerobics Center Longitudinal study," American Journal of Public Health, vol. 88, no. 12, pp. 1807-1813, 1998.

[12] M. L. Pollock and K. R. Vincent, Resistance Training for Health, Series 2, No. 8, The President's Council on Physical Fitness and Sports Research Digest, 1996.

[13] National Center for Chronic Disease Prevention and Health Promotion, Physical Activity and Health: A Report of the Surgeon General, US Deptartment of Health and Human Services, Atlanta, Ga, USA, 1996.

[14] C.-J. Liu and N. K. Latham, "Progressive resistance strength training for improving physical function in older adults," Cochrane Database of Systematic Reviews, no. 3, Article ID CD002759, 2009.

[15] R. Guthold, T. Ono, K. L. Strong, S. Chatterji, and A. Morabia, "Worldwide variability in physical inactivity: a 51-country survey," American Journal of Preventive Medicine, vol. 34, no. 6, pp. 486-494, 2008.

[16] S. A. Ham, J. Kruger, and C. Tudor-Locke, "Participation by US adults in sports, exercise, and recreational physical activities," Journal of Physical Activity and Health, vol. 6, no. 1, pp. 6-14, 2009.

[17] P. C. Hallal, L. B. Andersen, F. C. Bull et al., "Global physical activity levels: surveillance progress, pitfalls, and prospects," The Lancet, vol. 380, no. 9838, pp. 247-257, 2012.

[18] H.-J. F. Zunft, D. Friebe, B. Seppelt et al., "Perceived benefits and barriers to physical activity in a nationally representative sample in the European Union," Public Health Nutrition, vol. 2, no. 1 A, pp. 153-160, 1999.

[19] N. Thomas, E. Alder, and G. P. Leese, "Barriers to physical activity in patients with diabetes," Postgraduate Medical Journal, vol. 80, no. 943, pp. 287-291, 2004.

[20] A. Moschny, P. Platen, R. Klaaßen-Mielke, U. Trampisch, and T. Hinrichs, "Barriers to physical activity in older adults in Germany: a cross-sectional study," International Journal of Behavioral Nutrition and Physical Activity, vol. 8, article 121, 2011.

[21] D. Ding and E. Stamatakis, "Yoga practice in England 19972008: prevalence, temporal trends, and correlates of participation," BMC Research Notes, vol. 7, no. 1, article 172, 2014.

[22] G. Feuerstein, The Deeper Dimension of Yoga: Theory and Practice, Shambhala Publications, Boston, Mass, USA, 2003.

[23] S. Bryan, G. P. Zipp, and R. Parasher, "The effects of yoga on psychosocial variables and exercise adherence: a randomized, controlled pilot study," Alternative Therapies in Health and Medicine, vol. 18, no. 5, pp. 50-59, 2012.

[24] A. R. Kristal, A. J. Littman, D. Benitez, and E. White, "Yoga practice is associated with attenuated weight gain in healthy, middle-aged men and women," Alternative Therapies in Health \& Medicine, vol. 11, no. 4, pp. 28-33, 2005.
[25] M. Hagins, W. Moore, and A. Rundle, "Does practicing hatha yoga satisfy recommendations for intensity of physical activity which improves and maintains health and cardiovascular fitness?" BMC Complementary \& Alternative Medicine, vol. 7, article 40, 2007.

[26] M. D. Tran, R. G. Holly, J. Lashbrook, and E. A. Amsterdam, "Effects of hatha yoga practice on the health-related aspects of physical fitness," Preventive Cardiology, vol. 4, no. 4, pp. 165-170, 2001.

[27] V. S. Cowen and T. B. Adams, "Physical and perceptual benefits of yoga asana practice: results of a pilot study," Journal of Bodywork and Movement Therapies, vol. 9, no. 3, pp. 211-219, 2005.

[28] K. M. Chen, M. H. Chen, S. M. Hong, H. C. Chao, H. S. Lin, and C. H. Li, "Physical fitness of older adults in senior activity centres after 24-week silver yoga exercises," Journal of Clinical Nursing, vol. 17, no. 19, pp. 2634-2646, 2008.

[29] V. Gaurav, "Effects of hatha yoga training on the health related physical fitness," International Journal of Sports Science and Engineering, vol. 5, pp. 169-173, 2011.

[30] N. K. Patel, A. H. Newstead, and R. L. Ferrer, "The effects of yoga on physical functioning and health related quality of life in older adults: a systematic review and meta-analysis," The Journal of Alternative and Complementary Medicine, vol. 18, no. 10, pp. 902-917, 2012.

[31] J. Hill and A. Timmis, "Exercise tolerance testing," British Medical Journal, vol. 324, no. 7345, pp. 1084-1087, 2002.

[32] American College of Sports Medicine, Guildelines for Exercise Testing and Prescription, Lippincott Williams \& Wilkins, Philadelphia, Pa, USA, 8th edition, 2009.

[33] M. J. Buchfuhrer, J. E. Hansen, T. E. Robinson, D. Y. Sue, K. Wasserman, and B. J. Whipp, "Optimizing the exercise protocol for cardiopulmonary assessment," Journal of Applied Physiology: Respiratory Environmental \& Exercise Physiology, vol. 55, no. 5, pp. 1558-1564, 1983.

[34] G. F. Fletcher, G. Balady, L. H. Hartley, V. F. Froelicher, W. L. Haskell, and M. L. Pollock, "Exercise standards: a statement for healthcare professionals from the American Heart Association," Circulation, vol. 91, no. 2, pp. 580-615, 1995.

[35] G. Simoes, Mobile metronome (version 1.2.4.F), mobile application software, 2012, https://play.google.com/store/apps/ details?id=gabriel.metronome.

[36] S. S.-C. Hui and P. Y. Yuen, "Validity of the modified back-saver sit-and-reach test: a comparison with other protocols," Medicine \& Science in Sports \& Exercise, vol. 32, no. 9, pp. 1655-1659, 2000.

[37] S. C. Hui, W. C. Lau, W. K. Wong, P. Y. Yuen, and W. K. Lam, "Effect of a school-based intervention program on physical fitness of Chinese children with obesity: the fun-and-fit project," The ICHPER SD Journal of Research, vol. 3, pp. 6-11, 2008.

[38] C. L. Craig, A. L. Marshall, M. Sjöström et al., "International physical activity questionnaire: 12 -country reliability and validity," Medicine \& Science in Sports \& Exercise, vol. 35, no. 8, pp. 1381-1395, 2003.

[39] D. J. Macfarlane, C. C. Y. Lee, E. Y. K. Ho, K. L. Chan, and D. T. S. Chan, "Reliability and validity of the Chinese version of IPAQ (short, last 7 days)," Journal of Science and Medicine in Sport, vol. 10, no. 1, pp. 45-51, 2007.

[40] J. E. Ware Jr. and M. Kosinski, SF-36 Health Survey: Manual and Interpretation Guide, The Health Institute, New England Medical Center, Boston, Mass, USA, 1993. 
[41] C. L. K. Lam, B. Gandek, X. S. Ren, and M. S. Chan, "Tests of scaling assumptions and construct validity of the Chinese (HK) version of the SF-36 Health Survey," Journal of Clinical Epidemiology, vol. 51, no. 11, pp. 1139-1147, 1998.

[42] C. L. K. Lam, I. J. Lauder, T. P. Lam, and B. Gandek, "Population based norming of the Chinese (HK) version of the SF-36 health survey," Hong Kong Practitioner, vol. 21, no. 10, pp. 460-470, 1999.

[43] S. Sivasankaran, S. Pollard-Quintner, R. Sachdeva, J. Pugeda, S. M. Hoq, and S. W. Zarich, "The effect of a six-week program of yoga and meditation on brachial artery reactivity: do psychosocial interventions affect vascular tone?" Clinical Cardiology, vol. 29, no. 9, pp. 393-398, 2006.

[44] I. Devasena and P. Narhare, "Effect of yoga on heart rate and blood pressure and its clinical significance," International Journal of Biological \& Medical Research, vol. 2, pp. 750-753, 2011.

[45] L. Bernardi, G. Spadacini, J. Bellwon, R. Hajric, H. Roskamm, and A. W. Frey, "Effect of breathing rate on oxygen saturation and exercise performance in chronic heart failure," The Lancet, vol. 351, no. 9112, pp. 1308-1311, 1998.

[46] L. Bernardi, C. Porta, L. Spicuzza et al., "Slow breathing increases arterial baroreflex sensitivity in patients with chronic heart failure," Circulation, vol. 105, no. 2, pp. 143-145, 2002.

[47] C. N. Joseph, C. Porta, G. Casucci et al., "Slow breathing improves arterial baroreflex sensitivity and decreases blood pressure in essential hypertension," Hypertension, vol. 46, no. 4, pp. 714-718, 2005.

[48] D. C. Stanescu, B. Nemery, C. Veriter, and C. Marechal, "Pattern of breathing and ventilatory response to $\mathrm{CO}_{2}$ in subjects practicing hatha-yoga," Journal of Applied Physiology Respiratory Environmental and Exercise Physiology, vol. 51, no. 6, pp. 1625$1629,1981$.

[49] A. J. Bowman, R. H. Clayton, A. Murray, J. W. Reed, M. M. F. Subhan, and G. A. Ford, "Effects of aerobic exercise training and yoga on the baroreflex in healthy elderly persons," European Journal of Clinical Investigation, vol. 27, no. 5, pp. 443-449, 1997.

[50] P. R. Pullen, S. H. Nagamia, P. K. Mehta et al., "Effects of yoga on inflammation and exercise capacity in patients with chronic heart failure," Journal of Cardiac Failure, vol. 14, no. 5, pp. 407413, 2008.

[51] G. J. Salem, S. S. Y. Yu, M. Y. Wang et al., "Physical demand profiles of Hatha yoga postures performed by older adults," Evidence-Based Complementary and Alternative Medicine, vol. 2013, Article ID 165763, 29 pages, 2013.

[52] P. Tekur, C. Singphow, H. R. Nagendra, and N. Raghuram, "Effect of short-term intensive yoga program on pain, functional disability and spinal flexibility in chronic low back pain: a randomized control study," Journal of Alternative and Complementary Medicine, vol. 14, no. 6, pp. 637-644, 2008.

[53] P. E. Williams and G. Goldspink, "The effect of immobilization on the longitudinal growth of striated muscle fibres," Journal of Anatomy, vol. 116, no. 1, pp. 45-55, 1973.

[54] A. B. Moadel, C. Shah, J. Wylie-Rosett et al., "Randomized controlled trial of yoga among a multiethnic sample of breast cancer patients: effects on quality of life," Journal of Clinical Oncology, vol. 25, no. 28, pp. 4387-4395, 2007.

[55] C. Smith, H. Hancock, J. Blake-Mortimer, and K. Eckert, "A randomised comparative trial of yoga and relaxation to reduce stress and anxiety," Complementary Therapies in Medicine, vol. 15 , no. 2, pp. 77-83, 2007. 


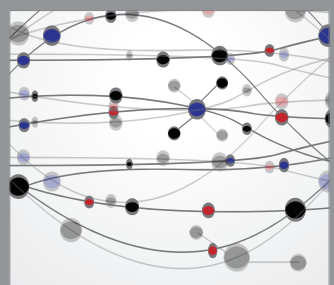

The Scientific World Journal
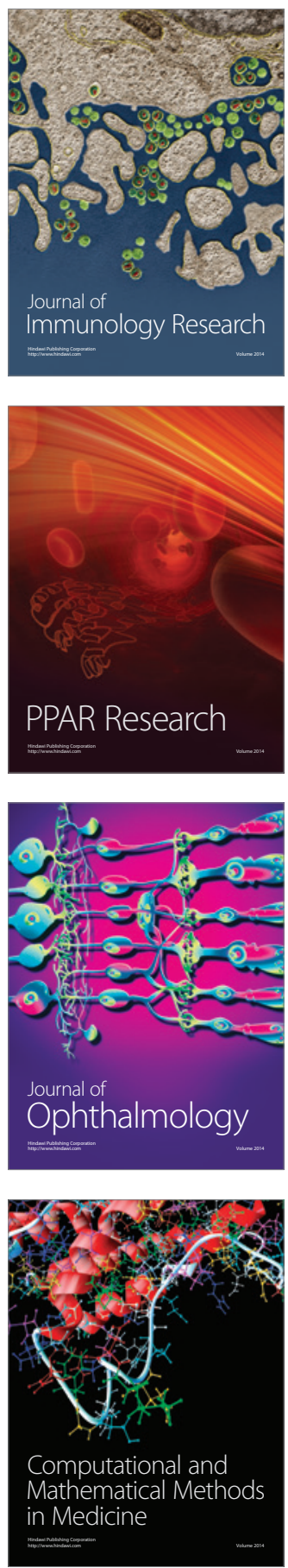

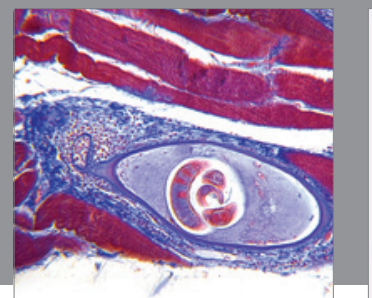

Gastroenterology

Research and Practice
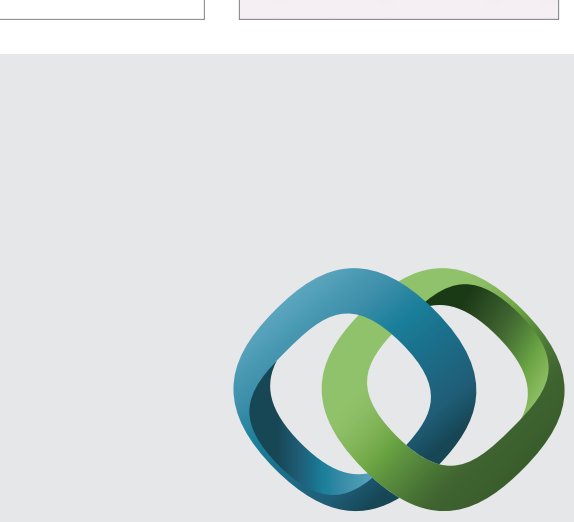

\section{Hindawi}

Submit your manuscripts at

http://www.hindawi.com
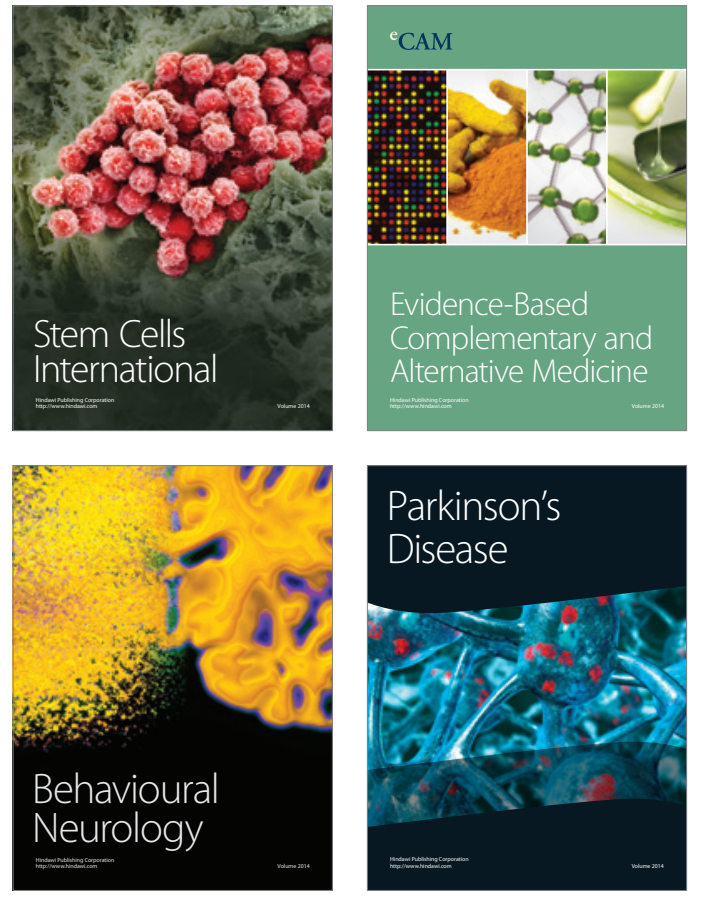
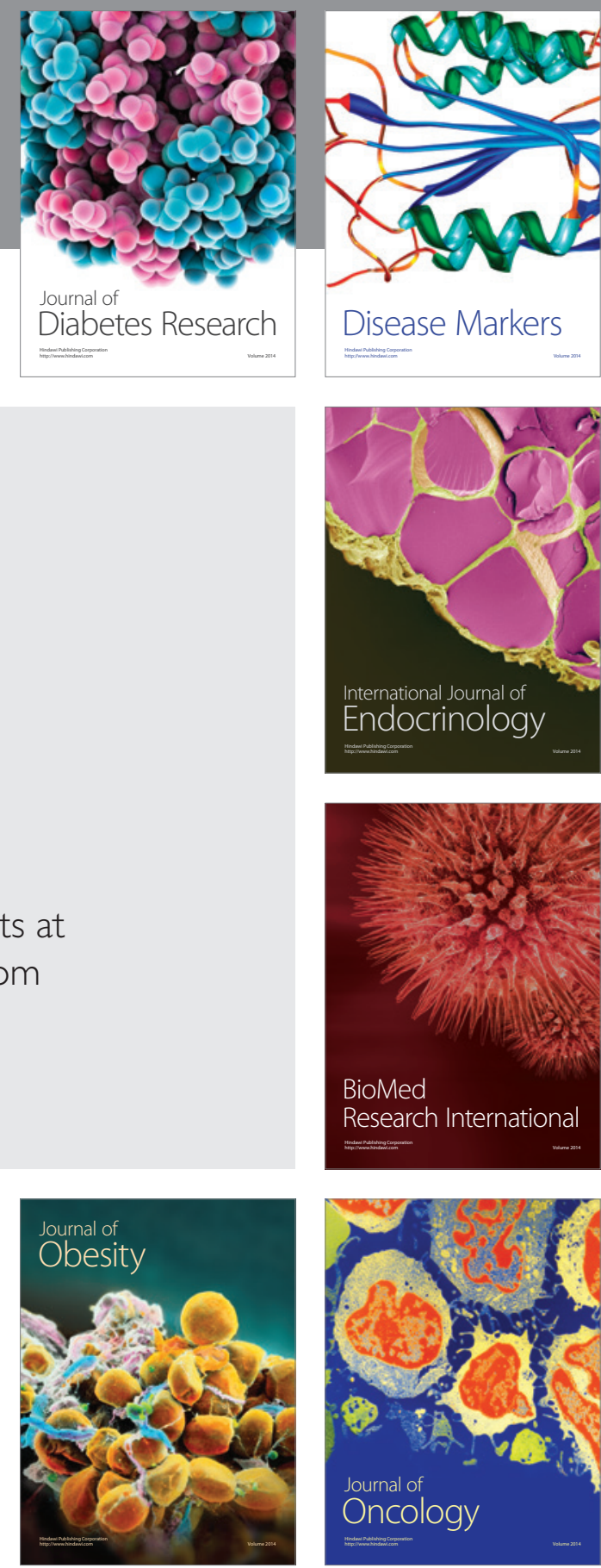

Disease Markers
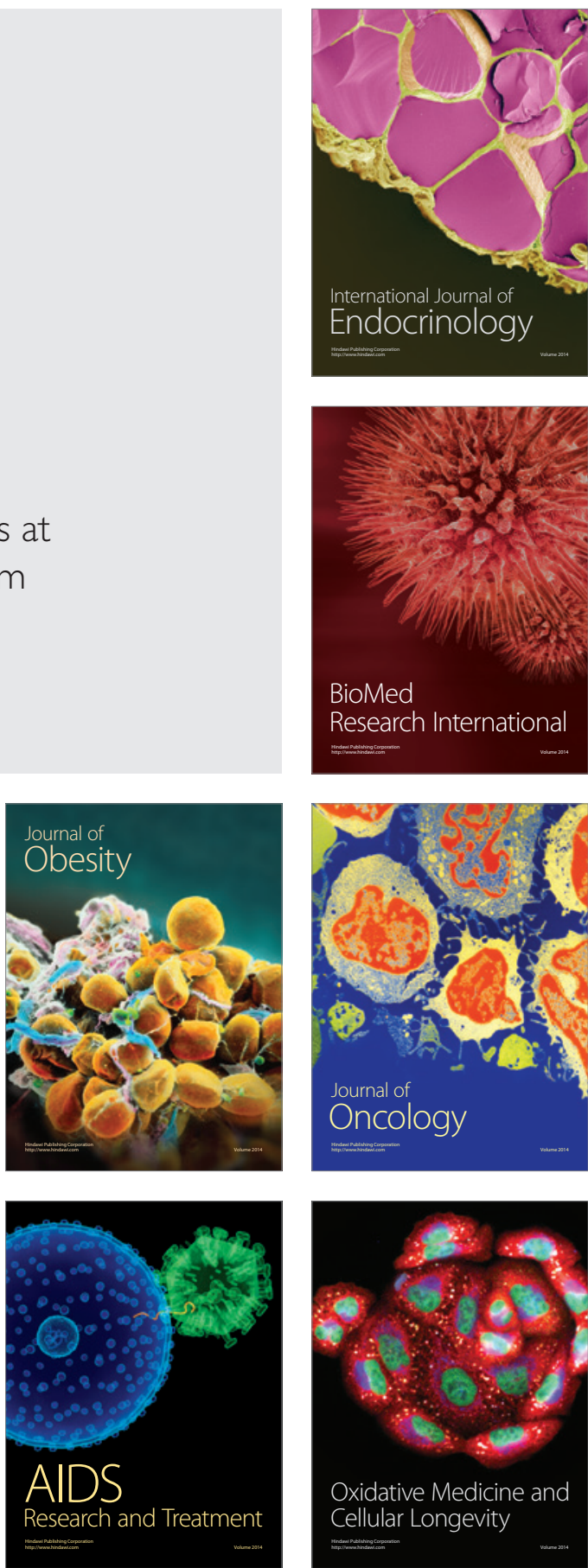